\title{
Evaluation of the Results Obtained from Microbiological Analysis of Blood Cultures over 5 Years
}

\author{
Mine Turhanoglu ${ }^{1}$, Esra Koyuncu ${ }^{1}$, Fulya Bayındır Bilman ${ }^{2}$, Arzu Onur $^{3}$, Fikret Tekay ${ }^{1}$ \\ ${ }^{1}$ Department of Medical Microbiology, Diyarbakır Selahaddin Eyyübi State Hospital, Diyarbakır, Turkey \\ ${ }^{2}$ Department of Medical Microbiology, İzmir Menemen State Hospital, İzmir, Turkey \\ ${ }^{3}$ Department of Medical Microbiology, Gazi Yaşargil Training and Research Hospital, Diyarbakır, Turkey
}

\author{
Email address: \\ mineturhanoglu@hotmail.com (M. Turhanoglu) \\ ${ }^{*}$ Corresponding author
}

\section{To cite this article:}

Mine Turhanoglu, Esra Koyuncu, Fulya Bayındır Bilman, Arzu Onur, Fikret Tekay. Evaluation of the Results Obtained from Microbiological Analysis of Blood Cultures over 5 Years. Journal of Family Medicine and Health Care. Vol. 2, No. 4, 2016, pp. 43-50. doi: $10.11648 /$ j.jfmhc.20160204.14

Received: August 24, 2016; Accepted: September 10, 2016; Published: November 3, 2016

\begin{abstract}
The infections caused by bacteria that reproduce in blood cultures are important medical problems that cause morbidity and mortality. The infections caused by resistant microorganisms are gradually increasing because of the patient's long stay in hospital, invasive procedures, and application of multi and parenteral antibiotic treatment. The microorganisms that reproduced in the blood cultures of patients in different cultures between 2010-2015 in Diyarbakır Selahaddin Eyyübi State Hospital and the resistance of these microorganisms to antibiotics were assessed retrospectively. In the study, a total of 196 patients' blood culture results were examined retrospectively. A total of $66.8 \%$ of the growth microorganisms (127) were composed of Gram positive cocci, 26.5\% of them (52) were composed of Gram-negative bacilli and $6.7 \%$ of them (11) were composed of Candida spp. Among the reproduced microorganisms, coagulase negative staphylococci (CNS) were found to be $52.5 \%$ (103), Staphylococcus aureus to be $4.9 \%$ (9), Acinetobacter spp to be $7.3 \%$ (14), Escherichia coli to be $4.7 \%$ (9), Klebsiella spp to be $8.4 \%$ (16), Candida spp. to be $6.7 \%$ (11), Pseudomonas spp. to be $4.7 \%$ (9), Enterococcus faecalis to be $2 \%$ (4), Micrococcus luteus to be $2 \%$ (4), Kocuria kristinae to be $2.5 \%$ (5), Rhizobium radiobacter to be $0.5 \%$ (1), Leuconostoc mesenteroides subsp. cremoris to be $1 \%$ (1), Sphingomonas paucimobilis $0.5 \%$ (1), Pantoea spp. to be $0.5 \%$ (1), and Stenotrophomonas maltophilia to be $0.5 \%$ (1). The highest rate of resistance was found to be against meropenem, imipenem and ceftazidime in Acinetobacter spp with $80 \%$, against ceftazidime in Klebsiella spp with $73.4 \%$, against imipenem with $75 \%$, against meropenem and ciprofloxacin with $62.5 \%$ in Pseudomonas aeruginosa, and against ceftriaxon, cefuroxime and cefuroxime axetil in Escherichia coli with $60 \%$. Penicillin with $100 \%$ and tetracycline with $33.3 \%$ in S. aureus; penicillin with $97.6 \%$ and erythromycin with $82.1 \%$ were the antibiotics to which the highest resistance developed. While no resistance was determined against fusidic acid, trimethoprim sulfamethoxazole, linezolid, vancomycin, teicoplanin, and tigecycline in $S$. aureus, the resistance was not determined only against tigecycline and vancomycin in CNS. Fifty seven $\%$ of $S$. aureus strains and $83.8 \%$ of CNS strains were found to be resistant to methicillin. In our study, it is aimed to determine the mostly reproduced bacteria in blood samples as the result of blood circulation infections of patients staying in different clinics and to research their resistance profiles that developed against antibiotics retrospectively.
\end{abstract}

Keywords: Blood Cultures, Antibiotic Sensitivity, S. aureus, Coagulase Negative Staphylococcus

\section{Introduction}

The infections caused by the bacteria that develop in blood cultures appear as a problem of public health that gives rise to morbidity and mortality. Susceptibility to the infections caused by resistant microorganisms is increasingly rising because of the long stay of the patients admitted to the hospital, intense invasive procedures, and application of multi and parenteral antibiotic treatment. The diversity of microorganisms and the increase in their rates of resistance cause problems in treatment and these infections progress 
with high mortality [1].

The infections caused by Gram negative bacteria are mostly opportunistic and are related to invasive procedures, mechanical ventilation, burn and surgical operations [2]. Pseudomonas aeruginosa bacteremia is an important cause fo hospital infections with high morbidity and mortality [3]. Candidemia, one of the invasive infections, is a severe clinical picture whose diagnosis and treatment are hard and which have rather high mortality. In our study, it is aimed to determine the mostly reproduced bacteria in blood samples as the result of blood circulation infections of patients staying in different clinics and to research their resistance profiles that developed against antibiotics retrospectively.

\section{Material and Methods}

The microorganisms reproduced from blood cultures of patients in different clinics between 01/01/2010 and $01 / 01 / 2015$ in our hospital and their resistance situations to various antibiotics were researched and assessed retrospectively. Blood culture samples, having completed 7day incubation period and giving " negative warning", from blood culture bottles followed with blood culture BACTEC 9050 (Becton Dickinson, USA) automatized blood culture system were assessed in terms of fake negativity by passaging to blood agar, and culture result was accepted to be negative. Those giving "positive warning" by the automatized system among the blood culture bottles were applied Gram strain and kept for 24 hours at $37^{\circ} \mathrm{C}$ by passaging to $5 \%$ blood agar, Eosine Methilene Blue Agar (EMB), chocolate agar, Sabouraud Dextrose Agar (SDA) media. Of the reproduced colonies, the identification and antibiotic susceptibility of microorganisms were determined by using VITEC version 2.0 (Biomerieux, France) system. Manual methods were utilized when needed.

\section{Results}

One of the samples reproducing the same bacterium taken from the right and the left arm of the same person and the samples contaminated by skin flora were excluded from the study; 169 isolates all belonging to different patients being included in the study. Sixty seven $\%$ of reproducing microorganisms (127) were composed of Gram positive coccus, $26.5 \%$ of them (52) of Gram negative bacilli and $6.7 \%$ of them (11) of Candida spp. Among the reproducing microorganisms, Coagulase Negative Staphylococcus (CNS) were found to be $52.5 \%$ (103), S. aureus to be $4.6 \%$ (9), Acinetobacter spp to be $7.3 \%$ (14), Escherichia coli to be $4.7 \%$ (9), Klebsiella spp to be $8.4 \%$ (16), Candida spp. to be $6.7 \%$ (11), Pseudomonas spp 4.7\% (9), Kocuria kristinae to be $2.5 \%$ (5), Enterococcus faecalis to be $2 \%$ (4), Micrococcus luteus 2\% (4), Rhizobium radiobacter to be $0.5 \%$ (1), Leuconostoc mesenteroides subsp. cremoris $1 \%$ (1), Sphingomonas paucimobilis to be $0.5 \%$ (1), Pantoea spp to be $0.5 \%$ (1), Stenotrophomonas maltophilia to be $0.5 \%$ (1) (Table 1).
Table 1. Distribution of growth bacteria from blood cultures between 20102015 .

\begin{tabular}{|c|c|c|c|c|c|}
\hline & & $\mathbf{n}$ & $(\%)$ & $\mathbf{n}$ & $\%$ \\
\hline \multirow{3}{*}{ Candida } & albicans & & & 7 & 63.6 \\
\hline & parapsilosis & 12 & $6.7 \%$ & 2 & 18.1 \\
\hline & tropicalis & & & 1 & 9 \\
\hline \multicolumn{2}{|c|}{ Pseudomonas aeruginosa } & 9 & $4.7 \%$ & & \\
\hline \multirow{9}{*}{ Staphylococcus } & aureus & & & 9 & 8 \\
\hline & capitis & & & 8 & 7.1 \\
\hline & epidermidis & & & 45 & 40 \\
\hline & haemolyticus & & & 12 & 10.7 \\
\hline & equorum & 117 & $58.9 \%$ & 1 & 0.9 \\
\hline & hominis & & & 35 & 31.2 \\
\hline & saprophyticus & & & 2 & 1.7 \\
\hline & scuri & & & 1 & 0.9 \\
\hline & warnerii & & & 4 & 3.5 \\
\hline \multicolumn{2}{|c|}{ Klebsiella pneumoniae } & 16 & $8.4 \%$ & \multirow{3}{*}{$\begin{array}{l}12 \\
2\end{array}$} & \multirow{3}{*}{$\begin{array}{l}85.7 \\
14.2\end{array}$} \\
\hline \multirow{2}{*}{ Acinetobacter } & baumannii & 14 & $73 \%$ & & \\
\hline & iwoffii & 14 & $1.5 \%$ & & \\
\hline \multicolumn{2}{|l|}{ Escherichia coli } & 9 & $4.7 \%$ & & \\
\hline \multicolumn{2}{|c|}{ Enterococcus faecalis } & 4 & $2 \%$ & & \\
\hline \multicolumn{2}{|c|}{ Micrococcus luteus } & 4 & $2 \%$ & & \\
\hline \multicolumn{2}{|l|}{ Kocuria kristinae } & 5 & $2.5 \%$ & & \\
\hline \multicolumn{2}{|c|}{ Rhizobium radiobacter } & 1 & $0.5 \%$ & & \\
\hline \multicolumn{2}{|c|}{ Leucostonococcus mesent cremoris } & 2 & $1 \%$ & & \\
\hline \multicolumn{2}{|c|}{ Sphingomonas paucimobilis } & 1 & $0.5 \%$ & & \\
\hline \multicolumn{2}{|l|}{ Pantoea spp } & 1 & $0.5 \%$ & & \\
\hline \multicolumn{2}{|c|}{ Stenotrophomonas maltophilia } & 1 & $0.5 \%$ & & \\
\hline \multicolumn{2}{|l|}{ Total } & 190 & & & \\
\hline
\end{tabular}

In staphylococcus, $S$. aureus was found to be $8 \%(9), S$. epidermidis to b $40 \%(45), S$. hominis to b $31.2 \%(35), \mathrm{S}$. haemolyticus to be $10.7 \%$ (12), S. equorum to be $0.9 \%$ (1), S. saprophyticus to be $1.7 \%$ (2), S. scuri to be $0.9 \%$ (1), $S$. warnerii to be $3.5 \%$ (4), S. capitis to be $7.1 \%$ (8); in Acinetobacter spp, A baumannii was found to be $85.7 \%$ (12), A. iwoffii to be $14.2 \%$ (2); in Candida spp, C. albicans was found to be $63.6 \%$ (7), C. parapsilosis to be $18.1 \%$ (2), C. tropicalis to be $9 \%$ (1) (Table 1). When the resistance profiles to antibiotics were examined, it was found that in staphylococcus, S. aureus was resistant to penicillin with $100 \%$ and to tetracycline with $33.3 \%$, CNS was resistant to penicillin with $97.6 \%$, and to erythromycin with $82.1 \%, S$. aureus strains were resistant to methicillin with $57.1 \%$ and CNS strains were resistant to methicillin with $83.8 \%$ (Table 2). All strains were found to be resistant to vancomycin. Resistance rates in $S$. aureus were found to be $33.3 \%$ for tetracyclin, $28.5 \%$ for rifampicin, $16.6 \%$ for erythromycin, ciprofloxacin, gentamicin, imipenem and clarithromycin, $14.2 \%$ for moxifloxacin, $57.1 \%$ for oxacillin, $14.2 \%$ for fosfomycin, $100 \%$ for penicillin. In $S$. aureus isolates, resistance to fusidic acid, trimethoprim/sulphametoxazole, linezolid, vancomycin, teicoplanin and tigecyclin was not detected (Table 2). In CNSs, tetracycline was found to be resistant by $61.7 \%$, rifampin by $51.2 \%$, erythromycin by $82.1 \%$, ciprofloxacin by $43.1 \%$, gentamicin by $12.2 \%$, imipenem by $\% 24.5$, clindamycin by $\% 18.8$, moxifloxacin by $39.5 \%$, fosfomycin by $65.6 \%$, penicillin, by $97.6 \%$, fusidic acid by $41.6 \%$, linezolid by $8.4 \%$, teicoplanin by $3.1 \%$, trimethoprim/sulfamethoxazole by $22.4 \%$ (Table 3 ). In CNS isolates, resistance to tigecycline and vancomycin 
was not found out.

Table 2. Antibiotic sensitivity rates of Staphylococcus aureus in blood culture between 2010-1015

\begin{tabular}{ll}
\hline & Sensitivity \% \\
\hline Ciprofloxacin & 83.3 \\
Gentamicin & 83.3 \\
Erytromicin & 83.3 \\
Imipenem & 83.3 \\
Moxifloxacin & 85.7 \\
Trimethoprim/sulfamethoxazole & 100 \\
Linezolid & 100 \\
Oxacillin & 42.8 \\
Fosfomicin & 85.7 \\
Tetracyclin & 66.6 \\
Fusidic acid & 100 \\
Rifampin & 71.4 \\
Penicillin & 0 \\
Tigecycline & 100 \\
Teicoplanin & 100 \\
Vancomycin & 100 \\
Clindamycin & 83.3 \\
\hline
\end{tabular}

For Pseudomonas aeruginosa, resistance rates were found to be $100 \%$ for amoxicillin/clavulanic acid and trimethoprim/sulphamethoxazole, $62,5 \%$ for ciprofloxacin, $75 \%$ for imipenem, $12.5 \%$ for meropenem, gentamicin, ceftazidime, $25 \%$ for piperacillin/tazobactam, $20 \%$ for cefepime; in $P$. aeruginosa, resistance to amikacin was not detected (Table 4).
Table 3. Antibiotic sensitivity rates of Coagulase Negative Staphylococcus in blood culture between 2010-1015.

\begin{tabular}{llll}
\hline & \multicolumn{3}{l}{ Coagulase Negative Staphylococcus } \\
\cline { 2 - 4 } & S \% & Intermediate \% & R \% \\
\hline Ciprofloxacin & 49 & 7.8 & 43.1 \\
Gentamicin & 77.1 & 10.5 & 12.2 \\
Erytromicin & 17.8 & & 82.1 \\
Imipenem & 24.5 & & 75.4 \\
Moxifloxacin & 60.4 & & 39.5 \\
Trimethoprim/sulfamethoxazole & 77.5 & & 22.4 \\
Linezolid & 91.5 & & 8.3 \\
Oksacillin & 16.1 & & 83.8 \\
Tetracycline & 38.2 & & 61.7 \\
Fusidic acid & 34.3 & 23.9 & 41.6 \\
Rifampin & 41.2 & 7.5 & 51.2 \\
Penicillin & 2.3 & & 97.6 \\
Tigecycline & 100 & & \\
Teicoplanin & 88.9 & & 11.1 \\
Vancomycin & 100 & & \\
Clindamycin & 78.2 & 2.8 & 18.8 \\
\hline
\end{tabular}

In Acinetobacter spp, $80 \%$ resistance developed against meropenem, imipenem, ceftazidime, $72.7 \%$ against cefoperazon, $60 \%$ against gentamicin, $50 \%$ against ampicillin /sulbactam, 25\% against levofloxacin and 18.1\% against ciprofloxacin (Table 4).

Table 4. Resistance rates of isolated Gram negative microorganisms to antimicrobials [(\%)].

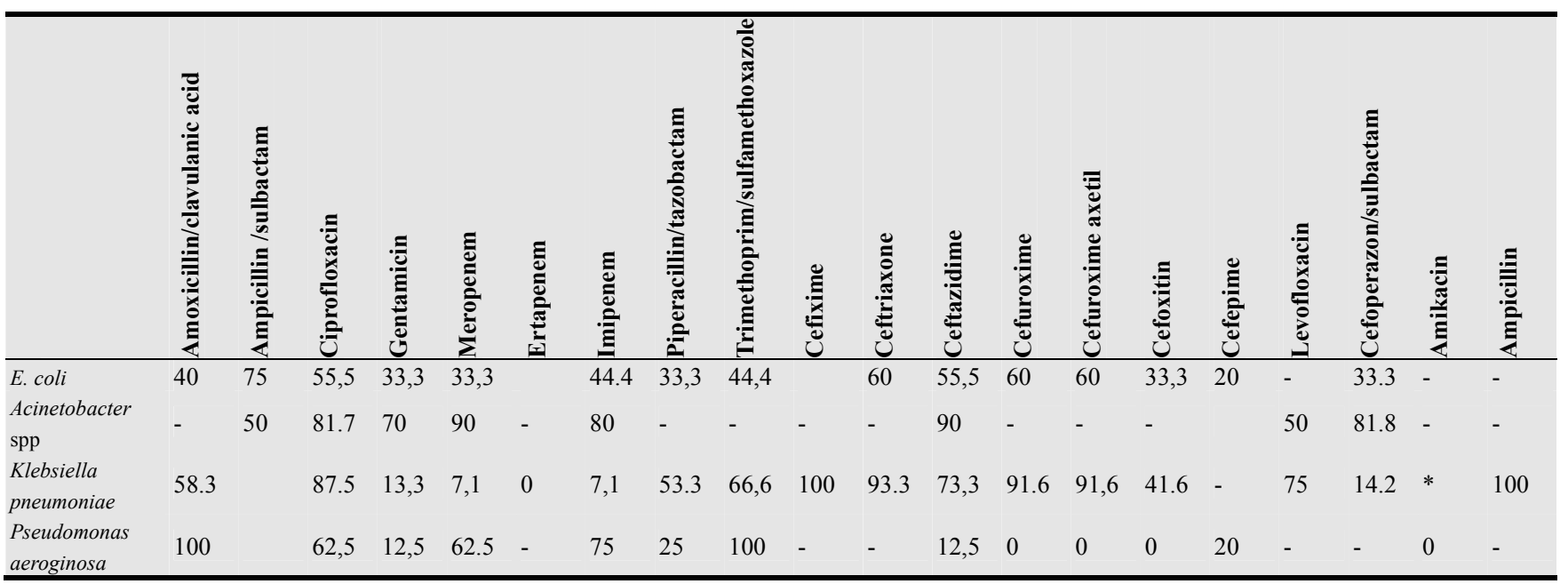

For Escherichia coli, it was observed that ceftriaxon, cefuroxime axetil and cefuroxime developed resistance by $60 \%$, ceftazidime and ciprofloxacin by $55.5 \%$, amoxicillin/ acid and cefepim by $20 \%$, trimethoprim/sulphamethoxazole by $44.4 \%$, ampicillin/sulbactam by $25 \%$, cefoxitin, cefoperazon/sulbactam, piperacillin/tazobactam, meropenem, gentamicin and imipenem by $33.3 \%$ (Table 4 ).

In Klebsiella pneumoniae, resistance was determined to be $91.6 \%$ to cefixim, cefuroxime axetil, cefuroxime, to be $73.3 \%$ to ceftazidime, $75 \%$ to ciprofloxacin, levofloxacin, $66.6 \%$ to trimethoprim/sulfamethoxazole, $63.6 \%$ to nitrofurantoine, $33.3 \%$ to cefoxitin, $13.3 \%$ to gentamicin, tazobactam/piperacillin, $8.3 \%$ to amoxicillin/clavulanic acid, $7.7 \%$ to ceftriaxon, $7.1 \%$ to meropenem, imipenem. In Klebsiella spp, resistance to ertapenem was not found (Table 4).

When the highest resistance rates of microorganisms to antibiotics were examined, it was observed that in Acinetobacter spp, $80 \%$ resistance was to meropenem, imipenem and ceftazidime; in Staphylococcus, S. aureus, $100 \%$ to penicillin, in CNS $97.6 \%$ to penicilline and $82.1 \%$ to erithtomycin, in Klebsiella spp 100\% to cefixim, $91.6 \%$ cefuroxim axetil and cefuroxime, in $P$. aeruginosa $100 \%$ to amoxicillin/clavulanic acid and trimethoprim /sulphamethoxazole, $75 \%$ to imipenem. In E. coli the highest 
resistance was to ceftariaxon, cefuroxime and cefuroxime axetil by $60 \%$, which is followed by ceftazidime by $55.5 \%$.

\section{Discussions}

Bacteremia and sepsis are clinical pictures with high morbidity and mortality that must be early diagnosed and treated [4]. According to the USA data, the rate of hospital stay due to bacteremia and sepsis has risen from 326000 up to 727000 in the last 10 years [5]. Blood circulation infections may cause several clinical pictures such as sepsis that threatens life with self-limiting infection, multiple organ failure, disseminated intravascular coagulopathy. Thus, it needs rapid and aggressive antimicrobial treatment [6]. Blood culture is commonly used for microorganisms that cause sepsis and bacteremia to be isolated and identified. Blood culture results are of great importance in revealing the infection factors, in directing to the accurate treatment by performing antibiotic susceptibility tests, and in reducing mortality. These microorganisms reproduced in blood cultures have a broad distribution. Although this is generally caused by Gram positive cocci and Gram negative bacilli, yeasts especially Candida species cause it effectively. Considering the studies on the issue; when Karakoç et al. examined blood culture results of 1 year, they isolated $67.4 \%$ Gram positive cocci, $15.6 \%$ Enterobacteriaceae, 3.6\% yeasts, and 3.4\% nonfermantative Gram negative bacteria. [7]. Yuce et al. found 59.3\% Gram negative bacteria, 28.1\% Gram positive bacteria, 12.5\% Candida spp [8], Çopur et al. found $80 \%$ Gram positive, 17\% Gram negative and 3\% Candida spp. [9]. In Kayseri, 64\% Gram positive cocci, 19\% Gram negative bacilli, and $8 \%$ Candida spp. was found [10], and Kuvat et al, in their study, stated that $48.5 \%$ of the microorganisms reproducing in blood cultures was composed of Gram positive, $47.5 \%$ of them was composed of Gram negative and $4 \%$ of them was composed of Candida spp. [11].

In a two-year study in Düzce, $64.4 \%$ Gram positive, $35.6 \%$ Gram negative bacteria were reproduced [12]. In a study carried out in Ankara, 337 were stated to be Gram positive, 78 to be Enterobacteriaceae and 18 to be Candida spp. [11]. Duman et al, in a study in which they assessed blood culture reproductions of on year, stated that th rate of Gram positive bacteria was $68.5 \%$, the rate of Gram negative ones was $31.5 \%$ [13].

In a study made in İzmir, $27.82 \%$ of bacteria having reproduced was Gram negative, $71.12 \%$ of them was Gram positive bacteria and $1.06 \%$ of them was C. albicans [14]. In another study made in İzmir again, 59\% Gram negative, $37.1 \%$ Gram positive bacteria and $3.9 \%$ fungi were determined [15]. The fact that bacteria profiles were found to be different in the same region from different hospitals made us think that one of the studies was based on the reproductions in blood cultures taken from intensive care unit patients. Considering the microorganisms reproducing in blood cultures abroad, Wasihun et al. determined that Gram positive was $68 \%$, Gram negative was $22.9 \%$ in one year period [16], Nwadioha et al. determined that $69.3 \%$ of patients with sepsis were Gram negative, and $30.7 \%$ of them were Gram positive [17].

In our study, 59.75 of microorganisms reproducing in blood culture were composed of Gram positive cocci, $26.5 \%$ of them were composed of Gram negative bacilli, and $6.7 \%$ of them were composed of Candida spp. When we looked at the studies on the issue, our study was observed to comply with the results from many parts of our country and from some centers abroad although Gram negative and Gram positive bacteria distributions had different percentages depending on regions.

In another study made in İzmir, among the factors isolated from the cultures with reproduction, A. baumannii was at the first rank with $21.5 \%$, Enterococcus spp. being found to be $17.4 \%, S$. aureus to be $12.1 \%, P$. aeruginosa to be $1.2 \%, K$. pneumoniae to be $8.8 \%$, CNS to be $8.4 \%$ and E. coli to be $7 \%$ [15]. In Kayseri, CNS was found to be $54 \%, S$. aureus and Acinetobacter to be \%, E.coli to be 5\% [10]. At the first rank among the microorganisms isolated in blood cultures by Er et al. there is $S$. aureus with $38.3 \%$, followed by CNS with $18.2 \%$, E. coli with $12.1 \%$, Enterococcus spp. with $7.3 \%, K$. pneumoniae with $7.1 \%, A$. baumannii with $4.8 \%, P$. aeruginosa with $4.1 \%$ and Candida spp with $3.3 \%$ [18]. The study of Kuvat et al. had a distribution of CNS with $34.8 \%$, Klebsiella spp with $12.8 \%$, E. coli with $2.6 \%, S$. aureus with $3 \%$, Candida spp with $3.7 \%$, Pseudomonas spp with $11.5 \%$, Acinetobacter spp. 9.6\%, Enterococcus spp. with 7.3\% [11].

In a study made in Düzce, $52.4 \%$ of Gram positive bacteria were identified to be CNS and $37.8 \%$ of them to be $S$. aureus and $7.4 \%$ of them to be Enterococcus; $36.9 \%$ of Gram negative bacteria were identified to be $E$. coli, $17.1 \%$ of them to be Klebsiella spp., $17.1 \%$ of them to be P. aeruginosa, $14.4 \%$ of them to be Enterobacter spp., $2.7 \%$ of them to be Acinetobacter spp. and $2.7 \%$ of them to be Stenotrophomonas maltophilia [12]. In the study of Yüce et al, $S$. aureus was at the first rank with $13.9 \%$, followed by Candida spp. with $12.5 \%$ and E.coli with $11.3 \%$ [8]. Willke determined in the studies that CNS and E.coli were the most frequently reproducing bacteria in blood cultures with $48 \%$ and $7 \%$, respectively [19].

Khorshed determined that in CNS isolates, $48.7 \%$ were $S$. hominis, $17.3 \%$ were $S$. haemolyticus, $3.3 \%$ were $S$. saprophyticus, $3.3 \%$ were $S$. simulans, $2.1 \%$ were $S$. warneri, $2.1 \%$ were $S$. chromogenes, $1.3 \%$ were $S$. eguorum, $1.3 \%$ were $S$. capitis and $0.7 \%$ were $S$. cohnii [24]. We, in our study, isolated $S$. aureus by $8 \%$, S. capitis by $7.1 \%, S$. epidermidis by $40 \%, S$. haemolyticus by $10.7 \%, S$. equorum by $0.9 \%, S$. hominis $31.2 \%, S$. saprophyticus by $1.7 \%, S$. scuri by $0.9 \%$ and S. warnerii by $3.5 \%$ in Staphylococcus.

Nwadioha et al. found that the most isolated bacteria were E. coli and $S$. aureus with $44.3 \%$ and $30.7 \%$, respectively [17]. Çopur et al. identified Gram negative bacteria as E. coli with $26 \%$, Klebsiella spp with $21.7 \%$, Pseudomonas spp with $21.7 \%$, Acinetobacter spp with $30.4 \%$, and identified Gram positive bacteria as coagulase negative staphylococcus with $87 \%$, S. aureus with $.7 \%$, and Enterococcus spp with 
9.3\% [9]. In another similar study made in Northern Ethiopia, it was found out that the distribution of bacteria reproducing in blood culture were $S$ aureus with $37.5 \%$, CNS with $30.6 \%$, E.coli with $3.1 \%$ [16].

In our retrospective study, among the reproducing microorgansims, Gram positives were found to be CNS (103) \%52.5, S. aureus (9) \%4.6, K. kristinae spp. (5) \%2.5, E. faecalis (4) \%2, M. luteus (4) \%2, L. mesenteroides subsp. cremoris (1)\%1; Gram negatives were found to be Klebsiella spp (16) \%8.4, Acinetobacter (14) \% 7.3, E. coli (9) \%4.7, Pseudomonas spp. (9) \%4.7, $R$. radiobacter(1) \%0.5, $S$. paucimobilis (1) \%0.5, Pantoea spp (1)\%0.5, S. maltophilia (1) $\% 0.5$. Candida spp. (11) was detected by $6.7 \%, C$. albicans (7) by $63.6 \%, C$. parapsilosis (2) by $18.1 \%$ and $C$. tropicalis (1) by $9 \%$. Distribution of species is shown in Table 1.

In the studies domestically, methicillin resistance was found in CNS by $56 \%, 70.2 \%, 42 \%, 91 \%, 75 \%, 79 \%$, respectively $[8,9,12,14,19,20]$, and in $S$. aureus strains by $69 \%, 50 \%, 25 \%, 34 \%$, respectively $[8,9,19,20]$. In the studies abroad, methicilline resistance was found in all $S$. aureus by $62.5 \%, 27 \%$ and $36 \%[16,21,22]$. In our study, $57.1 \%$ of $S$. aureus strains and $83.8 \%$ of CNS strains were found to be resistant to methicillin.

Considering the resistance to vancomycin, Mootsikapun et al. detected vancomycin resistance by $0.1-0.8 \%$ among MRSA isolates [21]. In a study made in Northern Ethiopia, all of the staphylococcus strains were found to be resistant to glycopeptides [16], but in the domestic studies vancomycin and teicoplanin resistance was not found in Staphylococcus strains $[8,9]$. All strains by Dokutan et al. were susceptible to vancomycin and teicoplanin, and $2.4 \%$ linezolid resistance was found [20]. Yilmaz et al. and Wasihun et al. did not report vancomycin resistance in Staphylococcus strains, and Khorsed et al, on the other hand, did not report vancomycin resistance in all CNS s isolated other than $S$. xylosus $[16,23$, 24]. In a study made in Kocaeli, teicoplanin resistance developed by $0.2 \%$ in CNS but was not encountered in $S$. aureus, vancomycin resistance was not found in CNS and $S$. aureus [19]. In our study, glycopeptide resistance was not seen among $S$. aureus.

In CNS, tetracyclin was found to be resistant by $61.7 \%$, rifampin by $51.2 \%$, erithromycin by $82.1 \%$, ciprofloxacin by $43.1 \%$, gentamicin by $12.2 \%$, imipenem by $24.5 \%$, clindamycin by $18.8 \%$, moxifloxacin by $39.5 \%$, fosfomycin by $65.6 \%$, penicillin by $97.6 \%$, fusidic acid by $41.6 \%$, clindamycin by $21.6 \%$, linezolid by $8.4 \%$, teicoplanin by $11.1 \%$, trimethoprim/sulfamethoxazole by $22.4 \%$. In CNSs vancomycin and tigecycline resistance was not found but teicoplanin and linezolid resistance was observed. The resistance rates in Staphylococcus were found to be $33.3 \%$ for tetracycline, $28.5 \%$ for rifampicin, $16.6 \%$ for erythromycin, ciprofloxacin, gentamicin, imipenem, and clindamycin, $14.2 \%$ for moxifloxacin, $14.2 \%$ for fosfomycin, $100 \%$ for penicillin. In $S$. aureus isolate, resistance to fusidic acid, trimethoprim/sulfamethoxazole, linezolid, vancomycin, teicoplanin, and tigecyclin was not detected.
Yilmaz et al. in Izmir, found the resistance in S. aureus with and without hospital infection by $100-71 \%$ in penicillin, $92-26 \%$ in erythromycin, $93-23 \%$ in clindamycin and levofloxacin, 33-16\% in trimetoprim/sulphametoxazole, 67$13 \%$ in fusidic acid, and the resistance in CNS by $100-83 \%$ in penicillin, $92-63 \%$ in erythromycin, $83-51 \%$ clindamycin, $83-40 \%$ in levofloxacin, $42-28 \%$ trimetroprim sulphametoxasol, $58-27 \%$ in fusidic acid [23].

16 of $35 \mathrm{CNS}$ strains isolated in another study made in 2010 in Portugal were found to be resistant to trimoxazole, 25 of them to ciprofloxacin, 19 of them to clindamycin [25]. Yet, in our study, resistance to fusidic acid, trimethoprim/sulphamethoxazole was not found for S.aureus. To other antibiotics, the same rate or more susceptibility was observed for CNS and S.aureus.

Köksal et al. found amoxicillin/clavulanic acid resistance to be $72 \%$ and $32 \%$ in Enterobacter-Klebsiella group and E. coli, Yüce et al. found it to be $64 \%$ in Klebsiella spp., $46 \%$ in E. coli $[8,26]$. In our study, amoxicillin/clavulanic acid resistance was found to be $58.3 \%$ in Klebsiella spp., $40 \%$ in E. coli.

Ceftriaxon resistance was reported to be $38 \%$ in E. coli, $84 \%$ in Pseudomonas spp. [27] and it was detected to be $73 \%$ in Acinerobacter spp. $46 \%$ in Pseudomonas spp [8]. In our study, ceftriaxon resistance was found to be $93.3 \%$ in Klebsiella spp and $60 \%$ in E.coli.

Resistance to meropenem did not develop in E. coli strains produced by Findık et al. and Köksal et al. while Yüce et al found the resistance to meropenem by $2 \%[8,26,27]$. Bektöre et al. did not find $E$. coli resistant to carbapenem but detected $16 \mathrm{~K}$. pneumoniae resistant to carbapenem [28]. In a study in İzmir, resistance to karbapanem was not found in Enterobacteriaceae members [14]. Carbapenem resistance was not seen in E. coli and Klebsiella spp strains reproducing in blood cultures, while resistance was detected in nonfermentative bacteria [23]. In our study, 4 E. coli were found to be resistant to imipenem and 3 to mereponem.

The resistance to meropenem in Pseudomonas spp was reported in the performed studies to be $12 \%, 25 \%, 24 \%$, $38.8 \%$ [8, 14, 27, 28]. In $P$. aeruginosa without hospital infection factor, resistance to meropenem was not seen, while resistance to imipenem was detected in one out of eight strains. Imipenem resistance was found in two out of five $P$. aeruginosa with nosocomial bacteremia factor, and meropenem resistance was found in three of them [23]. In our study, six out of eight Pseudomonas strains were found to be resistant to imipenem and six to meropenem.

In $A$. baumanni isolates, carbapenem resistance was found to be $66.7 \%, 85.75 \%$ in the involved studies $[14,28]$. Yüce et al. reported $2 \%$ imipenem resistance and Çopur et al. reported $85.7 \%$ [8,9]. Imipenem was found to be $56-74 \%$ and meropenem to be $50-71 \%$ in Kocaeli [19]. Al-Dorzi et al., in a six-year study, reported that bacteremia related to Acinetobacter spp are associated with strains resistant to multiple drugs [29]. In another study, almost half of $A$. baumannii strains were found to be resistant to carbapenems [23]. In our study, we found the resistance to carbapenem in 
Acinetobacter spp as $80 \%$ compatible with the studies.

Although in carbapenem resistance in Klebsiella isolates, Uzun et al. found imipenem as effective in all E. coli applied susceptibility tests and in $K$. pneumoniae not reproducing extended-spectrum beta lactamase (ESBL), they found resistance by $18 \%$ in $K$. pneumoniae strains reproducing ESBL [30]. Imipenem resistance in $K$. pneumoniae was found to be $1.3 \%$ [31]. When taken a look at carbapenem resistance in our study, it was found to be $7.1 \%$ in Klebsiella spp.

In Turkey data of Compact study, doripenem and meropenem show similar activities against Enterobacteriaceae, while imipenem was found to be four times as less active as them [31]. The most frequently isolated E. coli of Gram negative bacteria was found to be the most susceptible antimicrobial imipenem in Klebsiella spp. and Pseudomonas spp. [9].

Also, there are studies in which high resistance rates were followed as $72.6 \%$ in E. coli and $82.2 \%$ in K. pneumoniae for ciprofloxacin; besides a report for resistance was seen by $19 \%$ in a study [27] and $18 \%$ in another study in E. coli strains [26]. In Acinetobacter spp., 20\% [8] and 81\% [30] ciprofloxacin resistance was reported. In our study high rate of resistance to ciprofloxacin was found by $55.5 \%$ in $E$. coli, $81.7 \%$ in Acinetobacter spp and $82.5 \%$ in Klebsiella spp.

Ceftazidime resistance in Pseudomonas spp. in a study made in Elazı $\breve{g}$ was found to be $34 \%$ [8]; ceftazidime resistance ranging in $15-63 \%$ in Pseudomonas spp. was determined in the studies made in our country, while this rate was reported to be much higher in intensive care patients [8]. In our study we detected ceftazidime resistance $12.5 \%$. Resistance was reported to be by $2 \%$ for amikacin, $5 \%$ for piperacillin/tazobactam and $15 \%$ for ciprofloxacin in Pseudomonas spp. [8], by $28 \%$ [32], by $36 \%$ [33] and by $79.5 \%$ for ciprofloxacin [4]. In our study, no strains resistance to amikacin was found, while the resistance was obtained to be $25 \%$ to piperacillin/tazobactam, $62.5 \%$ to ciprofloxacin.

The incidence of ESBL was found to be $88.9 \%$ for $E$. coli, $56.2 \%$ for $K$. pneumoniae in our study. In the studies made in our country, ESBL positivity was detected to be $45.7 \%$ in $E$. coli, $67.8 \%$ in K. pneumoniae [28], $66.7 \%$ in E. coli, $74 \%$ in $K$. pneumoniae [14], $32 \%$ in E. coli strains and $38 \%$ in $K$. pneumoniae strains [30]. ESBL positivity by $47.3 \%$ was detected in K. pneumoniae isolated from blood cultures in a multi-centered widespread study [25].

In a study made abroad, it was stated that there was an increase in the infections caused by ESBL positive E. coli [35]. ESBL rates were found to be $43 \%$ and $45 \%$ in $E$. coli and Klebsiella strains without hospital infection factor, these rates were found to be $56 \%$ and $63 \%$, respectively in nosocomial bacteremia. ESBL reproduction of bacteria in hospital originated infections is a severe problem and ESBL reproduction in society originated infections as well is increasing day by day [36].

Candidemia, one of the invasive infections, is a severe clinic picture which is diagnosed and treated difficultly and which has a quite high mortality. An increase is observed in the incidence of Candida infections in parallel to the developments in diagnosis and treatment field through the increase in the number of patients receiving immunosuppressive treatment, in the usage of big surgical operations and broad spectrum antibiotics and in the patients whose general circumstances are disordered followed in intensive care units [37].

There are studies reporting that although the most common factor is C.albicans in Candidemia, the incidence of the species apart from $C$. albicans is gradually increasing. Gültekin et al., reproduced Candida in $0.48 \%$ [38] of 24709 blood cultures; $C$. albicans were detected to be by $23 \%, C$. parapsilosis to be $10 \%, C$. tropicalis to be $14 \%$. In a study made in Adana, C. parapsilosis by $33.9 \%$, C. albicans by $27.5 \%$, C. tropicalis by $16 \%$ were isolated [37], and in Kocaeli C. albicans by $50 \%$, C. tropicalis by $10.8 \%$, C. propsilosis by $21.7 \%$ were isolated [39]. In our study, Candida by $6 \%$ was reproduced ranging as $53.6 \% \quad C$. albicans, $18.1 \% \quad C$. parapsilosis, $9 \%$ C. tropicalis. Antifungal susceptibility of candida spp was not considered in this study. Other microorganisms reproducing in blood cultures are not scrutinized because of the insufficiency of their number.

\section{Conclusion}

Bacterial infections are frequently encountered problems in ICUs. Patients' having weak immune system and chronic disease, and the frequency of catheterization facilitates infection development. The diversity and antibiotic susceptibility of the bacteria isolated in blood cultures can differ according to geographical regions, hospital flora, antibiotics used in hospital and the profiles of the patients staying in hospitals. Therefore, each hospital should document the bacteria distribution and antibiotic susceptibility from time to time and establish treatment protocols according to these results. We are of the opinion that these results will guide especially in empirical treatment protocols of the clinician.

\section{Conflict of Interest}

The authors have declared that there is no conflict of interest and ethical adherence in this work.

\section{References}

[1] Mehli M., Gayyurhan E.D, Zer Y, Akgün S, Özgür Akın FE, Balc1 I. Microorganism isolated in blood cultures in the Gaziantep University hospital and their susceptibility to antibiotics. Infection Journal. 2007; 21: 141-5.

[2] Enoch DA, Birkett CI, Ludlam HA. Non-fermentative Gramnegative bacteria. Int J Antimicrob. Agents. 2007; 29: 33-1.

[3] Tang PC, Lee CC, Li CW, Li MC, Ko WC, Lee NY. Time-topositivity of blood culture: An independent prognostic factor of monomicrobial Pseudomonas aeruginosa bacteremia. J Microbiol Immunol Infect. 2015; 15: 1684-182. 
[4] Weinstein MP. Current blood culture methods and systems: clinical concepts, technology, and interpretation of results. Clin Infect Dis. 1996; 23: 40-6.

[5] Hall MJ, Williams SN, DeFrances CJ, Golosinskiy A. Inpatient care for septicemia or sepsis: A challenge for patients and hospitals. NCHS Data Brief. 2011; 62: 1-8.

[6] Negussie A, Mulugeta G, Bedru A, Ali I, Shimeles D, Lema T, Aseffa A. Bacteriological profile and antimicrobial susceptibility pattern of blood culture isolates among septicemia suspected children in selected hospitals Addis Ababa, Ethiopia. Int J Biol Med Re. 2015; 6: 4709-717.

[7] Karakoç AE, Ayyorgun Ş, Yücel M, Gündüz E. Microbiological Evaluation of an annual blood culture results. Dahili Tip Bilimleri Dergisi. 2006; 13: 2-6.

[8] Yüce P, Demirda K, Kalkan A, Özden M, Denk A, Kılıç SS. The Microorganisms Isolated from Blood Samples and their Susceptibility to Antibiotics. ANKEM Derg. 2005; 19(1): 171.

[9] Ç. Çiçek AŞ. Köksal Z, Ertürk A, Köksal E. Microorganisms isolated from blood cultures during the period of one year at the 82nd Year Rize State Hospital and their susceptibility to antibiotics. Turk Hij Den Biyol Derg. 2011; 68: 175-184.

[10] A.Altıntop Y, Karagöz S. Kayseri Traınıng Research Hospital Blood Culture Evaluation Results: Two-Year Results. 3. National Clinical Microbiology Congress.18-22 November 2015, Antalya, Turkey.

[11] Kuvat N, Nasuhbeyoğlu N, Özdemir B, Özdalgıçoğlu C, Esen A.B. Blood culture microorganisms are breeding in the intensive care unit at the inpatient and antibiotic sensitivity. 3. National Clinical Microbiology Congress, 18-22 November. 2015, Antalya, Turkey.

[12] Öksüz Ş, Yavuz T, Şahin I, Yıldırım M, Akgünođlu M, Kaya D, Öztürk E. Microorganisms Isolated from Blood Cultures and Susceptibility to antibiotics. Türk Mikrobiyol Cem Derg (2008) 38 (3-4): 117-121.

[13] Duman Y, Kuzucu Ç, Çuğlan SS. And antimicrobial susceptibility of bacteria isolated from blood cultures. Erciyes Tip Dergisi 2011; 33: 189-96.

[14] Ece G. The Evaluation of the Distribution and Antimicrobial Susceptibility Profile of the Strains Isolated from Blood Cultures. Med Bull Haseki 2013; 51: 151-56

[15] Çilli F, Soylu M, Aydemir Ş, Tünger A. Anesthesiology and Reanimation Clinic Intensive Care Unit at the hospitalacquired infections DISEASE Tagged: 2014 Year of Active Distribution and a variety of microorganisms are the antibiotic susceptibilities. 3. National Clinical Microbiology Congress. 18-22 November 2015. Antalya, Turkey.

[16] Wasihun AG, Wlekidan LN, Gebremariam SA, Dejene TA, Welderufael AL, et al. Bacteriological profile and antimicrobial susceptibility patterns of blood culture isolates among febrile patients in Mekelle Hospital, Northern Ethiopia. Springerplus. 2015; 4: 314-17.

[17] Nwadioha SI, Kashibu E, Alao OO, Aliyu I. Bacterial isolates in blood cultures of children with suspected septicaemia in Kano: a two-year study. Niger Postgrad Med J. 2011; 18: 130-33.

[18] Er H, Aşık G, Yoldaş Ö, Demir C, Keşli R. Determination of the Microorganisms Isolated from Blood Cultures and Their Antibiotic Susceptibility Rates Aim: Sepsis is a serious, lifethreatening condition that progresses. Türk Mikrobiyol Cem
Derg. 2015;45:48-4.

[19] Willke A, Azak E. Reproductive microorganisms from blood cultures and antibiotic sensitivity: Three-Year Results. ANKEM Derg. 2011;25 (Ek 1).

[20] Dokutan A, Hacıseyitoğlu D, Çă̆ Y, P.Yıldırım E, Batırel A, Özer S, Gönüllü N. The isolated from a clinical sample in Staphylococcus resistance to linezolid and Antibiotic Sensitivity. 3. Ulusal Klinik Mikrobiyoloji Congress. 18-22 Kasim 2015, Antalya, Turkey.

[21] Mootsikapun P, Trakulsomboon S, Sawanpanyalert P, Aswapokee N, Suankratay C. An overview of antimicrobial susceptibility patterns of Gram-positive bacteria from National Antimicrobial Resistance Surveillance Thailand (NARST) proGram from 2000 to 2005. J Med Assoc Thai. 2009; 92: 87-90.

[22] Rhee Y, Aroutcheva A, Hota B, Weinstein RA, Popovich KJ Evolving Epidemiology of Staphylococcus aureus Bacteremia. Infect Control Hosp Epidemiol. 2015; 36: 1417-422.

[23] Yılmaz N, Köse Ş, Ağuş N, Ece G, Akkoçlu G, Kıraklı C. Microorganisms Isolated from Blood Cultures of Intensive Care Unit Patients, their Antimicrobial Susceptibility and Etiological Agents in Nosocomial Bacteremia. ANKEM Derg 2010; 24: 12-9.

[24] Khorshed A, Özbal Y. Identification of Coagulase Negative Stphylococci Isolated From Blood Cultures and Determination and Investigation of Antibiotic Susceptibility. Sağlık Bilimleri Dergisi. 2012;21 153-63.

[25] F.Olivares M, H.Orozco R, Gutierrez RS, et al. Activity of daptomycin, ciprofloxacin, clindamycin and cotrimoxazole against coagulase-negative Staphylococcus strains with diminished susceptibility to vancomycin. Rev Esp Quimioter. 2010; 23: 81-6.

[26] Koksal F, Samast1 M: cases of resistance to antibiotics of enteric bacteria isolated from blood cultures. Klimik Derg. 2002; $15: 25-8$.

[27] Findik D, Tuncer, Ural O, Arslan U: Antibiotic Susceptibility of Gram-Negative Bacteria which are the causative agents of hospital infection. İnfeksiyon Derg. 2001; 15: 489-93.

[28] Bektöre B, Selek B M, Kula Atik T, Karakaş Ü, Sezer O, Baylan O.et al. A Training Research Features of the Isolated from Clinical Specimens Factors in Intensive Care in Hospital. 3. National Clinical Microbiology Congress. 18-22 November 2015. Antalya, Turkey.

[29] Al-Dorzi HM, Asiri AM, Shimemri A, Tamim HM, Al Johani SM, Al Dabbagh T, Arabi YM. Impact of empirical antimicrobial therapy on the outcome of critically ill patients with Acinetobacter bacteremia. Ann Thorac Med. 2015; 10: 256-62.

[30] K. Uzun B, Güngör SŞ, İlgün M, Özdemir R, Baran N, Ergin Ö.Y. The Frequency of Extended-spectrum Beta-lactamases and In-vitro Antibiotic Resistance in Escherichia coli and Klebsiella pneumoniae Strains Isolated from Blood Cultures. ANKEM Derg. 2012; 26: 181-86.

[31] Gür D, Gülay Z, Akan OA, Aktaş Z, Kayacan C.B.et ol, Cakici O, Eraç B, Gültekin M, Oğünç D, Söyletir G, Unal N, Uysal S. Resistance to newer beta-lactams and related ESBL types in Gram-negative nosocomial isolates in Turkish hospitals: results of the multicentre HITIT study. Mikrobiyol Bul. 2008; 42: 537-44. 
[32] Uzun B, Güngör S, Yurtsever S G, Afşar İ, Demirci M. Evalution of Resistance to Various Antibiotics in Pseudomonas aeruginosa and Acinetobacter baumannii Strains Isolated from Blood Cultures of Intensive Care Patients. ANKEM Derg 2012;26: 55-0.

[33] Ekincioğlu P, Perçin D. Antibiotic Susceptibilities of Clinical Pseudomonas aeruginosa Isolates. Journal of Health Sciences. 2013; 22: 141-49.

[34] Türk Dağı H, Arslan U, Findık D, Tuncer İ. Antibiotic Resistance Rates in Pseudomonas aeruginosa Strains Isolated from Blood Cultures. ANKEM Derg. 2011; 25: 107-10.

[35] Pitout JD, Laupland KB. Extended-spectrum beta- lactamaseproducing enterobacteriaceae: an emerging public health concern. Lancet Infect Dis. 2008; 8: 159-66.

[36] Ruppé E, Hem S, Lath S et al. CTX-M b-lactamases in
Escherichia coli from community-acquired urinary tract infections Cambodia. Emerg Infect Dis. 2009; 15: 741-8.

[37] Etiz Z P, Kibar F, Ekenoğlu Y, Yaman A. Retrospective Evaluation of Distribution and Antifungal Susceptibilities of Candida Species Isolated from Blood Cultures. ANKEM Derg. 2015; 29: 105-13.

[38] Gültekin B, Eyigör M, Telli M, Aksoy M, Aydın N. Retrospective Investigation of Candida Species Isolated from Blood Cultures during a Seven-year Period. ANKEM Derg. 2010; 24: 202-08.

[39] Keçeli Özcan S, Mutlu B, Dündar D, Willke A. Comparison of broth microdilution and E-test methods for the antifungal susceptibility testing of Candida spp. strains isolated from blood cultures. Mikrobiyol Bul 2010; 44: 263-71. 